\title{
Near-IR search for lensed supernovae behind galaxy clusters
}

\section{Implications for cluster modeling and cosmology ${ }^{\star}$}

\author{
T. Riehm ${ }^{1,2}$, E. Mörtsell ${ }^{2,3}$, A. Goobar²,3 , R. Amanullah²,3 , T. Dahlén ${ }^{4}$, J. Jönsson ${ }^{2,3}$, \\ M. Limousin ${ }^{5,6}$, K. Paech ${ }^{7}$, and J. Richard ${ }^{8,6}$
}

\author{
1 Department of Astronomy, Stockholm University, Albanova University Center, 10691 Stockholm, Sweden \\ e-mail: teresa@astro.su.se \\ 2 The Oskar Klein Centre, Stockholm University, 10691 Stockholm, Sweden \\ 3 Department of Physics, Stockholm University, Albanova University Center, 10691 Stockholm, Sweden \\ ${ }^{4}$ Space Telescope Science Institute, 3700 San Martin Drive, Baltimore, MD 21218, USA \\ 5 Laboratoire d'Astrophysique de Marseille, Université de Provence, CNRS, 38 rue Frédéric Joliot-Curie, \\ 13388 Marseille Cedex 13, France \\ ${ }^{6}$ Dark Cosmology Centre, Niels Bohr Institute, University of Copenhagen, Juliane Maries Vej 30, 2100 Copenhagen, Denmark \\ 7 Physikalisches Institut Universitat Bonn, Nussallee 12, 53115 Bonn, Germany \\ ${ }^{8}$ CRAL, Observatoire de Lyon, Université Lyon 1, 9 avenue Ch. André, 69561 Saint Genis Laval Cedex, France
}

Received 6 May 2011 / Accepted 27 September 2011

\section{ABSTRACT}

\begin{abstract}
Context. Massive galaxy clusters at intermediate redshifts act as gravitational lenses that can magnify supernovae ( $\mathrm{SNe}$ ) occurring in background galaxies.

Aims. We assess the possibility of using lensed SNe to constraint the mass models of galaxy clusters and the Hubble parameter at high redshift.

Methods. Owing to the standard candle nature of Type Ia supernovae (SNe Ia), observational information on the lensing magnification by an intervening galaxy cluster can be used to constrain the model for the cluster mass distribution. A statistical analysis using parametric cluster models was performed to investigate the possible improvements provided by lensed SNe Ia to the analysis of the accurately modeled galaxy cluster A1689 and the less well-modeled cluster A2204. Time delay measurements obtained from SNe lensed by accurately modeled galaxy clusters can be used to measure the Hubble parameter. For a survey of A1689, we estimate the expected rates of detectable SNe Ia and multiply imaged SNe.

Results. The velocity dispersion and core radius of the main cluster potential follow strong correlations with the predicted magnifications and can therefore be constrained by observations of SNe Ia in background galaxies. This technique proves especially powerful for galaxy clusters with only a few known multiple image systems. The main uncertainty in measurements of the Hubble parameter from the time delay of strongly lensed SNe is caused by cluster model uncertainties. For the extremely well-modeled cluster A1689, a single time delay measurement could be used to determine the Hubble parameter with a precision of $\sim 10 \%$.

Conclusions. Observations of SNe Ia behind galaxy clusters can be used to improve the mass modeling of the large-scale component of galaxy clusters and thus the distribution of dark matter. Time delays from SNe strongly lensed by accurately modeled galaxy clusters can be used to measure the Hubble constant at high redshifts.
\end{abstract}

Key words. cosmological parameters - galaxies: clusters: general - dark matter - supernovae: general - galaxies: halos gravitational lensing: strong

\section{Introduction}

Massive clusters have been successfully employed as gravitational telescopes (also known as Zwicky telescopes) to probe astronomical objects at very high redshifts (e.g., Kneib et al. 2004; Bradley et al. 2008; Bradač et al. 2009; Zheng et al. 2009). More recently, the use of near-IR observations to search for gravitationally magnified supernovae ( $\mathrm{SNe}$ ) behind massive galaxy clusters has been investigated. These data provide insight into the star formation rate at high redshifts, the progenitor systems

$\star$ Based on observations made with the NASA/ESA Hubble Space Telescope, obtained from the Data Archive at the Space Telescope Science Institute, which is operated by the Association of Universities for Research in Astronomy, Inc., under NASA contract NAS 5-26555. These observations are associated with programs \# 9134, 9289 and 10150 . of $\mathrm{SNe}$ and, if the cluster potential can be estimated properly, extend the supernova (SN) Ia Hubble diagram up to redshift $z \sim 3$ or possibly even higher. These measurements promise to dramatically extend the redshift baseline for which the expansion history of the universe can be probed, which could turn out to be essential for understanding the nature of dark energy.

A first transient object behind the massive cluster A1689 was found in a ground-based pilot survey at ESO using the ISAAC (Moorwood et al. 1998) camera on the VLT (Stanishev et al. 2009; Goobar et al. 2009, hereafter Paper I and Paper II, respectively). The transient was consistent with a reddened Type IIP SN at $z=0.59$ with a lensing magnification $\Delta m=$ 1.4 mag. Other SN candidates have been found in a survey with the newer HAWK-I (Pirard et al. 2004; Casali et al. 2006; Kissler-Patig et al. 2008) camera at VLT (Amanullah et al. 2011). Although not optimally cadenced for the purpose, 
HST detections of lensed SNe may also result from CLASH (Postman et al. 2011), which is part of the Multi-Cycle Treasury (MCT) program targeting 25 massive clusters, both at optical and near-IR wavelengths.

In this paper, we investigate the impact observations of gravitationally lensed $\mathrm{SNe}$ could have on the modeling of galaxy cluster potentials. We focus on the detection feasibility with either an 8-m class NIR survey such as the one with HAWK-I $\left(\mathrm{FOV} \approx 56 \operatorname{arcmin}^{2}\right)$, or with the Wide Field Camera 3 instrument on board the Hubble Space Telescope (HST/WFC3) with a ten times smaller FOV. Since SNe Ia have small intrinsic luminosity dispersion (after correction for light-curve shape and reddening), we can estimate the absolute magnification of the $\mathrm{SNe}$ and thus break the so-called mass-sheet degeneracy of gravitational lenses. This degeneracy implies that the density distribution of the lens can always be rescaled and a constant-density mass-sheet added such that the, also properly rescaled, source plane is projected onto the same observed images. Thereby, by breaking this degeneracy, direct constraints on the distribution of the dark matter component in galaxy clusters can be obtained.

If a $\mathrm{SN}$ is strongly lensed, a measurement of the time delay between the transient in the multiple images could potentially constrain the Hubble parameter (Refsdal 1964), thus both the dark matter and dark energy parameters (Goobar et al. 2002; Mörtsell \& Sunesson 2006; Suyu et al. 2010). Though not as precise as other cosmological tests for studying dark energy, the time delay technique has the major advantage of measuring cosmological parameters at redshifts where few other probes are currently available. Given the transient nature of $\mathrm{SNe}$, the time delay between multiple images could potentially be measured to very high precision. The main limitation of this technique is the strong degeneracy between the lens mass model and the Hubble parameter, $H_{0}$ (e.g., Wambsganss \& Paczynski 1994; Witt et al. 2000; Kochanek 2002; Zhao \& Qin 2003). However, observing cluster lenses with potentials well-constrained by a large number of already known multiple images or, in the case of a strongly lensed SNe Ia, direct magnification information, this degeneracy can be diminished (Oguri \& Kawano 2003). This technique is applicable to any object with a variable lightcurve that would make it possible to determine a time delay between different images, including quasars (QSOs) and gamma-ray bursts (GRBs). Although, there are $\sim 100$ strongly lensed QSOs currently known, time delays have been measured for only 20 of them (Oguri 2007). This is because most QSOs show variability only at a fairly low level, making time delay determinations challenging. Time delay measurements of QSOs behind cluster lenses have so far only been possible in two cases: SDSS J1029+2623 (three images, Inada et al. 2006) and SDSS J1004+4112 (five images, Fohlmeister et al. 2008). Image magnifications of about $\Delta m \sim 4.5$ mag have been recorded. However, the mass distributions in these clusters are not modeled well enough to constrain the Hubble constant. Although the time delay of a GRB can be determined with very high precision, they are expected to be too rare to admit a survey of lensed GRBs behind clusters. Therefore, in this paper we focus on time delays for multiply imaged $\mathrm{SNe}$.

The paper is structured as follows. In Sect. 2, we describe the cluster model used in our analysis. Section 3 investigates the observational prospects for lensed $\mathrm{SNe}$ behind a cluster. Constraints on the cluster modeling and the Hubble constant from lensed SNe are studied in Sects. 4 and 5. Finally, we discuss our results and conclude in Sect. 6.

Throughout the paper, we assume the cosmological parameters to be $\Omega_{\mathrm{M}}=0.3, \Omega_{\Lambda}=0.7$, and $H_{0}=70 \mathrm{~km} \mathrm{~s}^{-1} \mathrm{Mpc}^{-1}$.

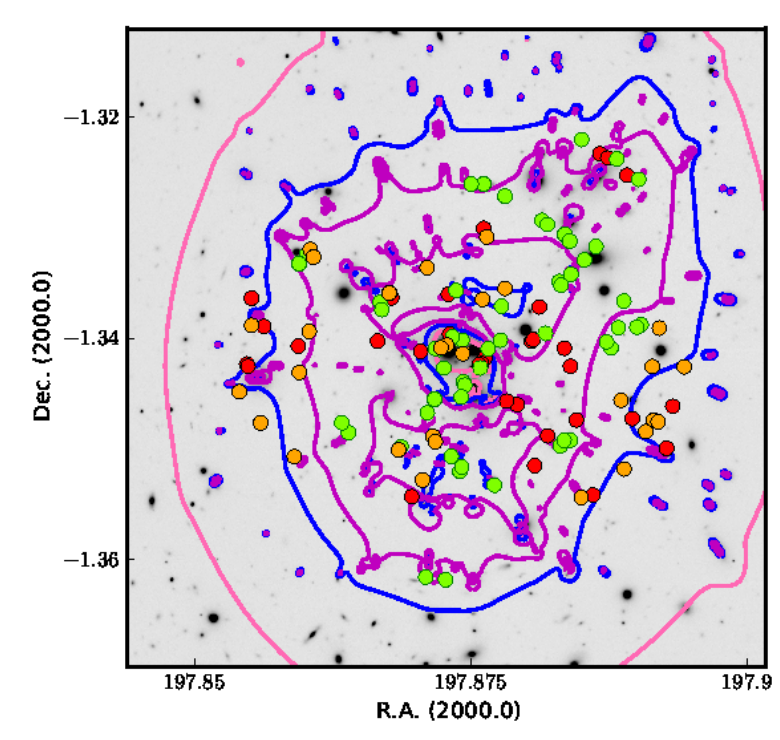

Fig. 1. Magnification map of A1689 overlaid on top of an HST ACS image of the cluster. The $\Delta m=1,2,3$ mag (pink, blue, magenta) contours are shown for a source at $z=2$. The green (orange) circles indicate the positions of strongly lensed galaxies with spectroscopic redshifts and time delays shorter than (above) five years. Red points indicate strongly lensed sources with photometric redshifts.

\section{Cluster modeling}

The focus of this paper is the massive cluster A1689 at redshift $z=0.187$, which is one of the most well-studied clusters. For this cluster, there are 34 known multiply imaged background galaxies and a total of 114 images. For 24 of the 34 background systems, there are secure spectroscopic redshifts available, ranging from $z=1.1$ to 4.9 (Broadhurst et al. 2005; Limousin et al. 2007) including a total of 82 images (see Fig. 1). For this cluster, a detailed mass model is available consisting of 272 parametrized pseudo-isothermal elliptical mass distributions (PIEMD). This is an updated version of the mass model presented in Limousin et al. (2007). Each potential is characterized using seven parameters, namely the center position (RA and Dec, given in arcseconds with respect to the BGC), the position angle $\theta$, and the parameters of the mass profile, i.e. the central velocity dispersion $\sigma$, the ellipticity $e$, the core radius $r_{\text {core }}$, and the cut radius $r_{\text {cut }}$. The model has 33 free parameters describing the mass distribution of the two large-scale dark matter clumps (clump 1 and clump 2), the dark matter halos of three individual galaxies that were found to play an essential role in producing multiply imaged systems (BCG, galaxy 1 and galaxy 2), and two scaling relations based on luminosity for the remaining identified cluster galaxies, $L^{*} r_{\text {cut }}$ and $L^{*} \sigma$ (compare Table 1$)$. These last two parameters are used to assign masses to the cluster galaxies, which are mainly early-type galaxies, via the scaling relations, $r_{\text {cut }}(L)=r_{\text {cut }}\left(L / L^{*}\right)^{1 / 2}$ and $\sigma(L)=\sigma\left(L / L^{*}\right)^{1 / 4}$, where $L^{*}$ is the luminosity of a typical galaxy in the cluster. The error bars given in Table 1 correspond to the $1 \sigma$ confidence level inferred from the MCMC optimization. Values in brackets are not optimized.

To investigate the power of lensed Type Ia SNe observations in constraining cluster models, we also study cluster A2204 at redshift $z=0.1524$. For this cluster, only one multiply lensed image system at $z=1.06$ is known, hence its mass distribution is far more weakly constrained than A1689. The mass model for this cluster consists of one main potential, describing the smooth cluster potential, and 34 cluster member galaxy potentials. All 
T. Riehm et al.: Near-IR search for lensed supernovae behind galaxy clusters. III.

Table 1. Parameter values inferred for the dark matter clumps and the scaling relation considered in the optimization procedure for galaxy cluster A1689.

\begin{tabular}{lccccccc}
\hline \hline Clump & RA (") & Dec (") & $e$ & $\theta$ & $r_{\text {core }}(\mathrm{kpc})$ & $r_{\text {cut }}(\mathrm{kpc})$ & $\sigma\left(\mathrm{km} \mathrm{s}^{-1}\right)$ \\
\hline Clump 1 & $0.5_{-0.2}^{+0.2}$ & $-8.5_{-0.4}^{+0.4}$ & $0.21_{-0.01}^{+0.01}$ & $90.4_{-1.1}^{+1.2}$ & $101.2_{-4.0}^{+4.6}$ & {$[1515.7]$} & $1445.5_{-14.7}^{+16.0}$ \\
Clump 2 & $-70.7_{-1.6}^{+1.3}$ & $49.1_{-3.6}^{+3.0}$ & $0.77_{-0.05}^{+0.04}$ & $78.6_{-2.6}^{+2.6}$ & $67.9_{-5.8}^{+8.0}$ & {$[501.0]$} & $647.1_{-4.9}^{+2.1}$ \\
BCG & $-1.1_{-0.2}^{+0.3}$ & $0.1_{-0.4}^{+0.4}$ & $0.48_{-0.04}^{+0.04}$ & $66.0_{-6.3}^{+6.9}$ & $5.3_{-1.1}^{+1.1}$ & $130.8_{-36.2}^{+37.2}$ & $441.7_{-1.1}^{+11.6}$ \\
Galaxy 1 & {$[49.1]$} & {$[31.5]$} & $0.70_{-0.15}^{+0.10}$ & $113.6_{-9.8}^{+7.2}$ & $25.6_{-3.4}^{+3.0}$ & $161.2_{-21.9}^{+13.3}$ & $262.8_{-16.6}^{+2.1}$ \\
Galaxy 2 & $-45.7_{-0.7}^{+0.4}$ & $31.6_{-0.9}^{+0.8}$ & $0.80_{-0.04}^{+0.05}$ & $45.5_{-2.1}^{+2.1}$ & $17.6_{-3.0}^{+1.8}$ & $182.8_{-9.7}^{+5.2}$ & $425.6_{-2.4}^{+2.5}$ \\
$L^{*}$ elliptical galaxy & $\cdots$ & $\cdots$ & $\cdots$ & $\cdots$ & {$[0.15]$} & $54.5_{-6.7}^{+3.7}$ & $154.6_{-3.4}^{+5.3}$ \\
\hline
\end{tabular}

Table 2. Parameter values inferred for the dark matter clump and the scaling relation considered in the optimization procedure for galaxy cluster A2204.

\begin{tabular}{lccccccc}
\hline \hline Clump & RA $\left({ }^{\prime \prime}\right)$ & Dec $\left({ }^{\prime \prime}\right)$ & $e$ & $\theta$ & $r_{\text {core }}(\mathrm{kpc})$ & $r_{\text {cut }}(\mathrm{kpc})$ & $\sigma\left(\mathrm{km} \mathrm{s}^{-1}\right)$ \\
\hline Clump 1 & {$[0.0]$} & {$[0.0]$} & $0.38_{0.14}^{+0.18}$ & $127.64_{6.63}^{+5.07}$ & $54.64_{22.34}^{+18.05}$ & {$[1000.0]$} & $933.47_{167.06}^{+160.66}$ \\
$L^{*}$ elliptical galaxy & $\cdots$ & $\cdots$ & $\cdots$ & $\cdots$ & {$[0.15]$} & {$[45]$} & $203.30_{28.94}^{+24.05}$ \\
\hline
\end{tabular}

potentials are described by PIEMD profiles and the model has a total of five free parameters: the ellipticity, $e$, position angle, $\theta$, core radius, $r_{\text {core }}$, and velocity dispersion, $\sigma$, of the main potential as well as the mass-luminosity scaling relation for the cluster galaxies, $L^{*} \sigma$ (compare Table 2, for details see Richard et al. 2010). The error bars given in Table 2 correspond to the $1 \sigma$ confidence level inferred from the MCMC optimization. Values in brackets are not optimized.

LENSTOOL is a software package for modeling the mass distributions of galaxies and clusters in the strong and weak lensing regime (Jullo et al. 2007) ${ }^{1}$. Monte Carlo Markov chains (MCMC) are used to constrain the free parameters of the cluster model derived from observational data of the background galaxies. The output contains a chain sampling the probability distribution of parameter values, each of which corresponds to a specific model realization. The density of parameter values gives a measure of the corresponding probability distribution for the parameters and can be used to estimate the errors in the corresponding parameters. For each realization, the magnification and time delay function at any given position behind the cluster, can be computed.

Assuming that we observe the magnification or time delay of a multiply imaged SN, we are able to rule out all realizations that do not agree with these (simulated) additional constraints. By comparing the remaining realizations to the original chain, we are then able to judge how powerful the additional constraints are in constraining the cluster potential.

\section{Supernovae behind clusters}

To investigate how efficient massive galaxy clusters are in facilitating the detection of distant $\mathrm{SNe}$ and improving the constraints on cluster modeling or cosmology, we need a robust estimate of how many SNe behind the cluster can be expected during a given survey time. For example, for a five year monthly survey of one very massive A1689-like cluster with HAWK-I, it has been shown that the total number of SNe expected in the background galaxies is on the order of 40-70 SNe, out of which approximately 20-30 are SNe Ia (depending on the underlying rates estimate for the various SN types, see Paper II).

\footnotetext{
${ }^{1}$ http://www.oamp.fr/cosmology/lenstool/
}

For the purpose of studying time delays using galaxy cluster A1689, we focus primarily on potential SN explosions in the 24 known multiply lensed background galaxies with spectroscopic redshifts. Ten multiply imaged galaxies with only photometric redshifts are not taken into consideration to avoid additional sources of error caused by uncertainties in the photometric redshifts. Furthermore, as more data are collected, new multiple image systems might be detected. The numbers we present below should therefore be considered as lower limits.

Since the rate of SNe is expected to be coupled to the starformation rate (SFR) in these galaxies, we use rate predictions for the strongly lensed systems derived from local estimates of the SFR. Since the UV luminosity is dominated by the most short-lived stars, it is closely related to star formation. Thus, we use $L_{2800}$, the flux at rest-frame $\lambda_{\text {eff }}=2800 \AA$ (redshifted to optical bands) as a tracer of the SFR in the strongly lensed galaxies. Since there are very deep HST observations of A1689 with multiple filters, these estimates are remarkably precise.

The absolute $L_{2800}$ magnitude is derived after taking into account the distance modulus, K-corrections, extinction, and lensing magnification from the cluster model. Finally, we use the relation between $L_{2800}$ and SFR from Dahlén et al. (2007) to relate the flux to star formation

$\operatorname{SFR}\left(M_{\odot} \mathrm{yr}^{-1}\right)=L_{2800}\left(\mathrm{erg} \mathrm{s}^{-1} \mathrm{~Hz}^{-1}\right) \times\left(7.0 \times 10^{27}\right)^{-1}$

The expected rate for core collapse $\mathrm{SNe}$ is calculated from

$r_{\mathrm{CC}}(z)=k_{8}^{50} \times \operatorname{SFR}(z)$,

where $k_{8}^{50}=0.007 M_{\odot}^{-1}$ is estimated using a Salpeter IMF and a progenitor mass range of between 8 and 50 solar masses. For $\mathrm{SNe}$ Ia, we assume that the rate has two components, one proportional to the SFR and one proportional to stellar mass, according to the model of Scannapieco \& Bildsten (2005). The rate is given by

$r_{\mathrm{Ia}}(t)=A \operatorname{SFR}(t)+B M(t)$.

The mass, $M$, of individual galaxies are calculated using massto-light ratios from Bell et al. (2003). The resulting rates for core collapse $\mathrm{SNe}$ and $\mathrm{SNe}$ Ia in multiply lensed background galaxies with spectroscopic redshifts and predicted time delays shorter than five years are summarized in Table 3. In total, we 
Table 3. All image pairs of multiply lensed background galaxies with spectroscopic redshift and a predicted time delay shorter than five years.

\begin{tabular}{|c|c|c|c|c|c|c|c|c|c|c|c|c|c|}
\hline \multirow[t]{2}{*}{ Images } & \multirow[t]{2}{*}{$z_{\text {spec }}$} & \multirow{2}{*}{$\begin{array}{r}\Delta t^{a} \\
(\mathrm{~d})\end{array}$} & $\Delta m_{1}$ & $\Delta m_{2}{ }^{b}$ & $r_{\mathrm{CC}} \times 10^{2}$ & $r_{\text {Ia }} \times 10^{3 c}$ & \multicolumn{7}{|c|}{ Detectable SN types ${ }^{d}$} \\
\hline & & & \multicolumn{2}{|c|}{ (mag) } & \multicolumn{2}{|c|}{$\left(\mathrm{yr}^{-1}\right)$} & Ia & IIP & IIL & $\mathrm{IIL}_{\mathrm{b}}$ & IIn & $\mathrm{Ib} / \mathrm{c}$ & $\mathrm{HN}$ \\
\hline $1.1+1.2$ & 3.04 & $-83.2_{-69.4}^{+76.9}$ & $3.80_{-0.29}^{+0.30}$ & $5.58_{-0.74}^{+1.21}$ & $2.07_{1.04}^{+1.07}$ & $1.32_{0.66}^{+0.68}$ & $\mathrm{y}$ & $\mathrm{y}$ & $\mathrm{y}$ & $\mathrm{y}$ & $\mathrm{y}$ & $\mathrm{y}$ & $\mathrm{y}$ \\
\hline $1.3+1.6$ & 3.04 & $1248.2_{-148.9}^{+139.5}$ & $2.12_{-0.05}^{+0.05}$ & $0.88_{-0.07}^{+0.08}$ & $\ldots$ & $\ldots$ & $\mathrm{y}$ & & & & $\mathrm{y}$ & & \\
\hline $1.4+1.5$ & 3.04 & $-139.4_{-79.7}^{+87.7}$ & $2.85_{-0.06}^{+0.07}$ & $1.88_{-0.04}^{+0.05}$ & $\ldots$ & $\ldots$ & $\mathrm{y}$ & & & $\mathrm{y}$ & $\mathrm{y}$ & & $\mathrm{y}$ \\
\hline $2.2+2.3$ & 2.53 & $-163.0_{-72.2}^{+77.7}$ & $2.97_{-0.06}^{+0.07}$ & $2.01_{-0.04}^{+0.05}$ & $1.74_{0.85}^{+0.85}$ & $1.12_{0.53}^{+0.52}$ & $\mathrm{y}$ & & $\mathrm{y}$ & $\mathrm{y}$ & $\mathrm{y}$ & $\mathrm{y}$ & $\mathrm{y}$ \\
\hline $2.4+2.5$ & 2.53 & $1072.8_{-132.0}^{+119.4}$ & $2.07_{-0.05}^{+0.05}$ & $1.00_{-0.07}^{+0.07}$ & $\ldots$ & $\ldots$ & $\mathrm{y}$ & & & $\mathrm{y}$ & $\mathrm{y}$ & & $\mathrm{y}$ \\
\hline $4.1+4.2$ & 1.16 & $575.6_{-64.9}^{+63.9}$ & $2.96_{-0.04}^{+0.04}$ & $2.85_{-0.05}^{+0.05}$ & $0.44_{0.16}^{+0.16}$ & $0.28_{0.10}^{+0.10}$ & $\mathrm{y}$ & $\mathrm{y}$ & $\mathrm{y}$ & $\mathrm{y}$ & $\mathrm{y}$ & $\mathrm{y}$ & $\mathrm{y}$ \\
\hline $5.1+5.2$ & 2.64 & $-31.2_{-16.6}^{-17.6}$ & $3.54_{-0.08}^{+0.08}$ & $2.82_{-0.07}^{+0.07}$ & $0.97_{0.43}^{+0.43}$ & $0.65_{0.27}^{+0.27}$ & $\mathrm{y}$ & $\mathrm{y}$ & $\mathrm{y}$ & $\mathrm{y}$ & $\mathrm{y}$ & $\mathrm{y}$ & $\mathrm{y}$ \\
\hline $6.1+6.2$ & 1.15 & $70.8_{-170.4}^{+166.5}$ & $2.81_{-0.04}^{+0.08}$ & $2.95_{-0.09}^{+0.12}$ & $0.45_{0.21}^{+0.21}$ & $6.51_{5.50}^{+5.50}$ & $\mathrm{y}$ & $\mathrm{y}$ & $\mathrm{y}$ & $\mathrm{y}$ & $\mathrm{y}$ & $\mathrm{y}$ & $\mathrm{y}$ \\
\hline $6.1+6.3$ & 1.15 & $1759.3_{-249.5}^{+237.3}$ & $2.81_{-0.04}^{+0.04}$ & $3.69_{-0.44}^{+0.74}$ & $\ldots$ & $\ldots$ & $\mathrm{y}$ & $\mathrm{y}$ & $\mathrm{y}$ & $\mathrm{y}$ & $\mathrm{y}$ & $\mathrm{y}$ & $\mathrm{y}$ \\
\hline $6.1+6.4$ & 1.15 & $222.8_{-161.3}^{+145.5}$ & $2.81_{-0.04}^{+0.04}$ & $3.30_{-0.33}^{+0.56}$ & $\ldots$ & $\ldots$ & $\mathrm{y}$ & $\mathrm{y}$ & $\mathrm{y}$ & $\mathrm{y}$ & $\mathrm{y}$ & $\mathrm{y}$ & $\mathrm{y}$ \\
\hline $6.2+6.3$ & 1.15 & $1680.8_{-250.1}^{+262.3}$ & $2.96_{-0.10}^{+0.11}$ & $3.69_{-0.44}^{+0.034}$ & $\ldots$ & $\ldots$ & $\mathrm{y}$ & $\mathrm{y}$ & $\mathrm{y}$ & $\mathrm{y}$ & $\mathrm{y}$ & $\mathrm{y}$ & $\mathrm{y}$ \\
\hline $6.2+6.4$ & 1.15 & $157.3_{-119.9}^{+113.0}$ & $2.95_{-0.10}^{+0.11}$ & $3.30_{-0.33}^{+0.56}$ & $\ldots$ & $\ldots$ & $\mathrm{y}$ & $\mathrm{y}$ & $\mathrm{y}$ & $\mathrm{y}$ & $\mathrm{y}$ & $\mathrm{y}$ & $\mathrm{y}$ \\
\hline $6.3+6.4$ & 1.15 & $-1526.1_{-286.3}^{+281.8}$ & $3.73_{-0.44}^{+0.73}$ & $3.31_{-0.32}^{+0.56}$ & $\ldots$ & $\ldots$ & $\mathrm{y}$ & $\mathrm{y}$ & $\mathrm{y}$ & $\mathrm{y}$ & $\mathrm{y}$ & $\mathrm{y}$ & $\mathrm{y}$ \\
\hline $7.2+7.3$ & 4.87 & $645.0_{-82.3}^{+96.0}$ & $1.77_{-0.07}^{-0.06}$ & $0.31_{-0.13}^{+0.32}$ & $13.04_{6.08}^{+6.06}$ & $8.34_{3.71}^{+3.70}$ & & & & & & & \\
\hline $10.2+10.3$ & 1.83 & $1376.2_{-148.6}^{+14.0}$ & $2.49_{-0.08}^{+0.08}$ & $0.16_{-0.12}^{+0.12}$ & $5.42_{2.47}^{+2.48}$ & $4.31_{1.57}^{+1.58}$ & $\mathrm{y}$ & & & $\mathrm{y}$ & $\mathrm{y}$ & & $\mathrm{y}$ \\
\hline $12.2+12.3$ & 1.83 & $63.1_{-12.9}^{+13.0 .0}$ & $3.69_{-0.06}^{+0.06}$ & $7.03_{-0.61}^{+1.05}$ & $0.68_{0.32}^{+0.32}$ & $0.42_{0.20}^{+0.20}$ & $\mathrm{y}$ & $\mathrm{y}$ & $\mathrm{y}$ & $\mathrm{y}$ & $\mathrm{y}$ & $\mathrm{y}$ & $\mathrm{y}$ \\
\hline $14.1+14.2$ & 3.40 & $-236.8_{-17.2}^{+14.1}$ & $4.76_{-0.25}^{+0.39}$ & $2.92_{-0.06}^{+0.06}$ & $\ldots$ & $\ldots$ & $\mathrm{y}$ & & & $\mathrm{y}$ & $\mathrm{y}$ & & $\mathrm{y}$ \\
\hline $15.1+15.3$ & 1.82 & $888.6_{-139.3}^{+138.8}$ & $2.61_{-0.08}^{+0.08}$ & $0.74_{-0.13}^{+0.14}$ & $0.91_{0.42}^{+0.42}$ & $0.56_{0.26}^{+0.26}$ & $\mathrm{y}$ & & & $\mathrm{y}$ & $\mathrm{y}$ & & $\mathrm{y}$ \\
\hline $17.1+17.2$ & 2.66 & $-78.8_{-20.8}^{+24.6}$ & $3.21_{-0.15}^{+0.14}$ & $1.40_{-0.09}^{+0.09}$ & $15.13_{6.19}^{+6.21}$ & $10.92_{3.82}^{+3.84}$ & $\mathrm{y}$ & & & $\mathrm{y}$ & $\mathrm{y}$ & & $\mathrm{y}$ \\
\hline $19.1+19.5$ & 2.60 & $1507.0_{-92.0}^{+208.8}$ & $2.40_{-0.06}^{+0.06}$ & $1.02_{-0.10}^{+0.11}$ & $3.58_{1.53}^{+1.53}$ & $2.43_{0.96}^{+0.96}$ & $\mathrm{y}$ & & & $\mathrm{y}$ & $\mathrm{y}$ & & $\mathrm{y}$ \\
\hline $19.3+19.4$ & 2.60 & $-84.1_{-12.7}^{+10.5}$ & $3.11_{-0.09}^{+0.06}$ & $5.14_{-0.18}^{+0.21}$ & $\ldots$ & $\ldots$ & $\mathrm{y}$ & $\mathrm{y}$ & $\mathrm{y}$ & $\mathrm{y}$ & $\mathrm{y}$ & $\mathrm{y}$ & $\mathrm{y}$ \\
\hline $22.1+22.2$ & 1.70 & $462.2_{-98.7}^{+111.6}$ & $2.21_{-0.05}^{+0.09}$ & $0.13_{-0.20}^{-0.18}$ & $2.77_{1.06}^{+1.03}$ & $2.16_{0.70}^{+0.68}$ & $\mathrm{y}$ & & & $\mathrm{y}$ & $\mathrm{y}$ & & $\mathrm{y}$ \\
\hline $24.2+24.3$ & 2.63 & $-1029.2_{-148.9}^{+157.2}$ & $4.81_{-0.61}^{+1.14}$ & $2.96_{-0.05}^{+0.05}$ & $0.93_{0.42}^{+0.43}$ & $0.62_{0.26}^{+0.27}$ & $\mathrm{y}$ & $\mathrm{y}$ & $\mathrm{y}$ & $\mathrm{y}$ & $\mathrm{y}$ & $\mathrm{y}$ & $\mathrm{y}$ \\
\hline $24.2+24.4$ & 2.63 & $-1364.2_{-196.9}^{+198.6}$ & $4.81_{-0.61}^{+1.14}$ & $2.83_{-0.08}^{+0.09}$ & $\ldots$ & $\ldots$ & $\mathrm{y}$ & $\mathrm{y}$ & $\mathrm{y}$ & $\mathrm{y}$ & $\mathrm{y}$ & $\mathrm{y}$ & $\mathrm{y}$ \\
\hline $24.3+24.4$ & 2.63 & $\begin{array}{c}-196.9 \\
-343.0_{-164.7}^{+169.7}\end{array}$ & $2.96_{-0.05}^{+0.01}$ & $2.83_{-0.08}^{+0.08}$ & $\ldots$ & $\ldots$ & $\mathrm{y}$ & $\mathrm{y}$ & $\mathrm{y}$ & $\mathrm{y}$ & $\mathrm{y}$ & $\mathrm{y}$ & $\mathrm{y}$ \\
\hline $29.2+29.3$ & 2.57 & $1017.8_{-170.9}^{+16.5}$ & $3.04_{-0.05}^{+0.05}$ & $4.90_{-0.66}^{+1.27}$ & $0.71_{0.37}^{+0.40}$ & $0.53_{0.26}^{+0.27}$ & $\mathrm{y}$ & $\mathrm{y}$ & $\mathrm{y}$ & $\mathrm{y}$ & $\mathrm{y}$ & $\mathrm{y}$ & $\mathrm{y}$ \\
\hline $29.2+29.4$ & 2.57 & $136.7_{-140.0}^{+174.9}$ & $3.04_{-0.05}^{+0.05}$ & $2.78_{-0.08}^{+0.00}$ & $\ldots$ & $\ldots$ & $\mathrm{y}$ & $\mathrm{y}$ & $\mathrm{y}$ & $\mathrm{y}$ & $\mathrm{y}$ & $\mathrm{y}$ & $\mathrm{y}$ \\
\hline $29.3+29.4$ & 2.57 & $-881.6_{-168.9}^{+179.0}$ & $4.90_{-0.66}^{+1.27}$ & $2.78_{-0.07}^{+0.08}$ & $\ldots$ & $\ldots$ & $\mathrm{y}$ & $\mathrm{y}$ & $\mathrm{y}$ & $\mathrm{y}$ & $\mathrm{y}$ & $\mathrm{y}$ & $\mathrm{y}$ \\
\hline $30.1+30.2$ & 3.05 & $-27.3_{-191.8}^{+186.6}$ & $2.71_{-0.07}^{+0.09}$ & $4.89_{-0.52}^{+0.99}$ & $3.41_{2.44}^{+2.48}$ & $2.40_{1.65}^{+1.68}$ & $\mathrm{y}$ & & & $\mathrm{y}$ & $\mathrm{y}$ & & $\mathrm{y}$ \\
\hline $30.1+30.3$ & 3.05 & $1022.6_{-316.4}^{+339.4}$ & $2.71_{-0.08}^{+0.09}$ & $4.86_{-0.70}^{+1.10}$ & $\ldots$ & $\ldots$ & $\mathrm{y}$ & & & $\mathrm{y}$ & $\mathrm{y}$ & & $\mathrm{y}$ \\
\hline $30.2+30.3$ & 3.05 & $1052.7_{-166.8}^{+183.1}$ & $4.89_{-0.53}^{+0.08}$ & $4.86_{-0.70}^{+1.09}$ & $\ldots$ & $\ldots$ & $\mathrm{y}$ & $\mathrm{y}$ & $\mathrm{y}$ & $\mathrm{y}$ & $\mathrm{y}$ & $\mathrm{y}$ & $\mathrm{y}$ \\
\hline $32.1+32.2$ & 3.00 & $350.1_{-91.6}^{+90.7}$ & $2.15_{-0.06}^{+0.05}$ & $2.97_{-0.07}^{+0.07}$ & $4.38_{1.75}^{+1.75}$ & $3.07_{1.07}^{+1.07}$ & $\mathrm{y}$ & & & $\mathrm{y}$ & $\mathrm{y}$ & & $\mathrm{y}$ \\
\hline $32.3+32.4$ & 3.00 & $759.0_{-98.9}^{+98.0}$ & $2.19_{-0.05}^{+0.06}$ & $1.29_{-0.05}^{+0.06}$ & $\ldots$ & $\ldots$ & $\mathrm{y}$ & & & $\mathrm{y}$ & $\mathrm{y}$ & & \\
\hline $35.1+35.3$ & 1.91 & $1246.8_{-95.9}^{+95.9}$ & $2.69_{-0.06}^{+0.06}$ & $0.97_{-0.14}^{-0.05}$ & $2.54_{1.07}^{+1.07}$ & $1.65_{0.67}^{+0.67}$ & $\mathrm{y}$ & & & $\mathrm{y}$ & $\mathrm{y}$ & & $\mathrm{y}$ \\
\hline $36.1+36.2$ & 3.01 & $-45.4_{-21.0}^{+18.2}$ & $4.92_{-0.16}^{+0.17}$ & $4.52_{-0.14}^{+0.13}$ & $0.06_{0.03}^{+0.03}$ & $0.04_{0.02}^{+0.02}$ & $\mathrm{y}$ & $\mathrm{y}$ & $\mathrm{y}$ & $\mathrm{y}$ & $\mathrm{y}$ & $\mathrm{y}$ & $\mathrm{y}$ \\
\hline
\end{tabular}

Notes. Errors are given as $1 \sigma$ confidence intervals. ${ }^{(a)}$ Predicted time delay between the images. A negative time delay indicates that image 2 will occur before image 1. ${ }^{(b)}$ Predicted magnification for image 1 and image 2, respectively. ${ }^{(c)}$ Rate estimates for core collapse SNe and SNe Ia, respectively. ${ }^{(d)}$ Detectability of a lensed SN for the different SN types assuming a limiting magnitude of 25.25 mag (Vega) in F140W, e.g. in a survey using one-third of an orbit with the HST/WFC3 instrument (compare with Fig. 2).

expect $\sim 0.6$ core collapse $\mathrm{SNe}$ and $\sim 0.05 \mathrm{SNe}$ Ia per year to explode in the known multiply imaged galaxies with spectroscopic redshifts.

To calculate the properties of the lens systems, i.e., masses and SFRs, we use available observations in the HST/ACS optical filters (f435w, f475w, f555w, f625w, f775w, f850lp), as well as HST/NICMOS IR filters (f110w, f160w) from Richard et al. (in prep.).

Using the cluster model to calculate magnifications, we investigate the observability of these systems. To be observable, not only must the time delay be shorter than the survey duration (here assumed to be five years) but the luminosity of both images must also lie above the observation threshold. Here we have chosen a magnitude limit of 25.25 mag (Vega) in F140W, corresponding to what could be achieved, e.g., during one-third of an orbit with the HST/WFC3. In Fig. 2, the predicted light curves for SNe of Type Ia and IIP are shown. As can be seen, nearly all SNe Ia exploding in one of the known multiply lensed background galaxies would be bright enough to be observable, while about half of the SNe IIP, mostly at lower redshift and with high magnification, would lie above the luminosity threshold. Columns 8-14 in Table 3 indicate the SN type that would be observable for each image combination producing appropriate time delays.

\section{Cluster model constraints}

When modeling the potential of a cluster as described in Sect. 2, information on the position of the multiple images is used to optimize the free parameters. In general, the addition of a constant surface density $\kappa_{\mathrm{c}}$ to a lens potential leaves the resulting image positions of a system unchanged. This is known as the mass sheet degeneracy. In the case of A1689, owing its large number of known multiply imaged systems at different redshifts, this 
T. Riehm et al.: Near-IR search for lensed supernovae behind galaxy clusters. III.

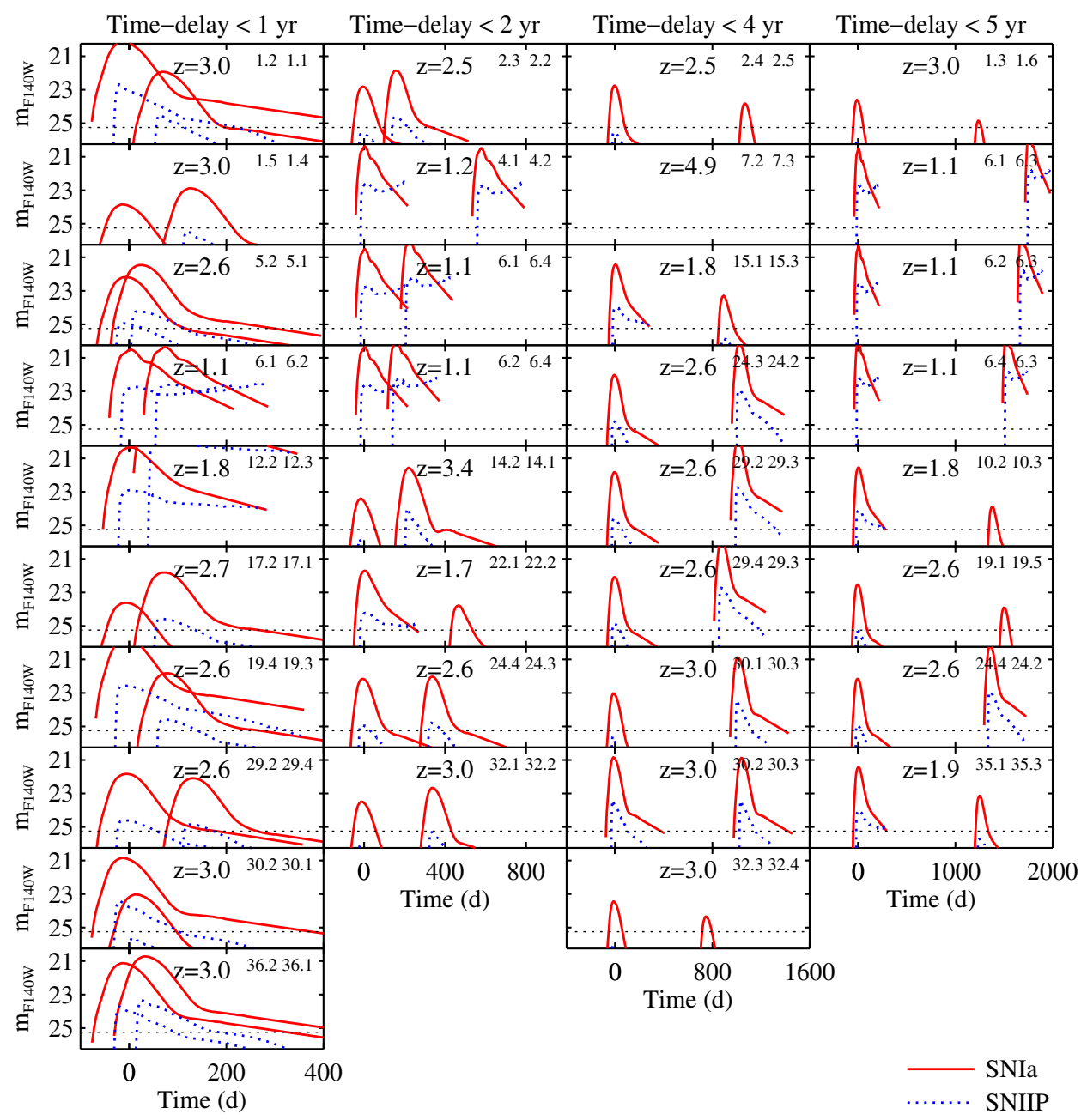

Fig. 2. Simulated light curves of SNe Ia and IIP for pairs of images of strongly lensed galaxies behind A1689. Only pairs with time delays shorter than five years are shown. Additional information on different image pairs labeled by, e.g., 1.2 and 1.1 in the top right corner of each sub panel, can be found in Table 3. The dashed line indicates an expected magnitude limit 25.25 (Vega) in F140W.

degeneracy can be broken. However, for galaxy clusters with very few observed lensed systems, this degeneracy can be problematic. The detection of a SN Ia behind a cluster offers a direct way of constraining the lens potential. Owing to the standard candle nature of SNe Ia (see Goobar \& Leibundgut 2011, for a recent review) the information on the absolute magnitude can be used to measure the magnification at the position of the $\mathrm{SN}$ and compare it to mass model predictions. One would then be able to rule out realizations producing deviant magnifications. The precision of the magnification measurements are ultimately limited by the intrinsic scatter in the brightness of SNe Ia after corrections for light-curve shape and color, about $0.1 \mathrm{mag}$ in the rest-frame optical wavelength region (Conley et al. 2011). We do not anticipate any additional sources of error caused by the cluster in the line of sight. For instance, observing at near-IR wavelengths ensures that corrections from dust in the (low-z) cluster are small, especially since galaxy clusters are relatively dust-free environments (see Dawson et al. 2009, and references therein). Thus, a SN Ia exploding in any of the background galaxies behind the cluster could be used to place constraints on the lensing potential. As discussed in Paper II, we expect to detect 20-30 SNe Ia (depending on the underlying rates estimates) to be detectable, e.g., in a five year monthly survey at VLT. To assess the power of this method, we investigate the strength of the correlation between the optimized model parameters and the magnification for different positions behind the cluster.
Figure 3 shows the absolute value of the correlation coefficients for the predicted magnification and the free input parameters as a function of position for an image at redshift $z=2$. The parameters shown are those describing the two dark matter clumps (clump 1 and clump 2) and the cluster galaxy scaling relations $\left(L^{*} r_{\text {cut }}\right.$ and $\left.L^{*} \sigma\right)$ in the model of A1689. A value close to 1 implies a very strong correlation, while a correlation coefficient close to 0 implies no correlation. As can be seen in Fig. 3, the free parameters differ in terms of the strength of their correlation with the predicted magnification both with each other and the position behind the cluster. The dependence of the correlation strength on redshift is very weak. In general, among all 33 free optimized parameters, the ones that show the strongest correlations with magnification and therefore the possibility of improving the model once a SN Ia is observed, are the ellipticity, $e$, core radius, $r_{\text {core }}$, and velocity dispersion, $\sigma$, of the main lensing potential (clump 1). Other parameters, such as the corresponding parameters for the cluster galaxies and the position of the main lensing potentials display a weaker correlation and can probably not be more tightly constrained using SN Ia observations in the case of A1689.

Figure 4 shows an example of how the model parameters could be constrained if the magnification of an image is known to a precision of $0.1 \mathrm{mag}$. In this example, we consider a SN Ia observed in the multiply-lensed image 18.1 with spectroscopic redshift $z=1.82$ under the most favorable conditions. Since this 

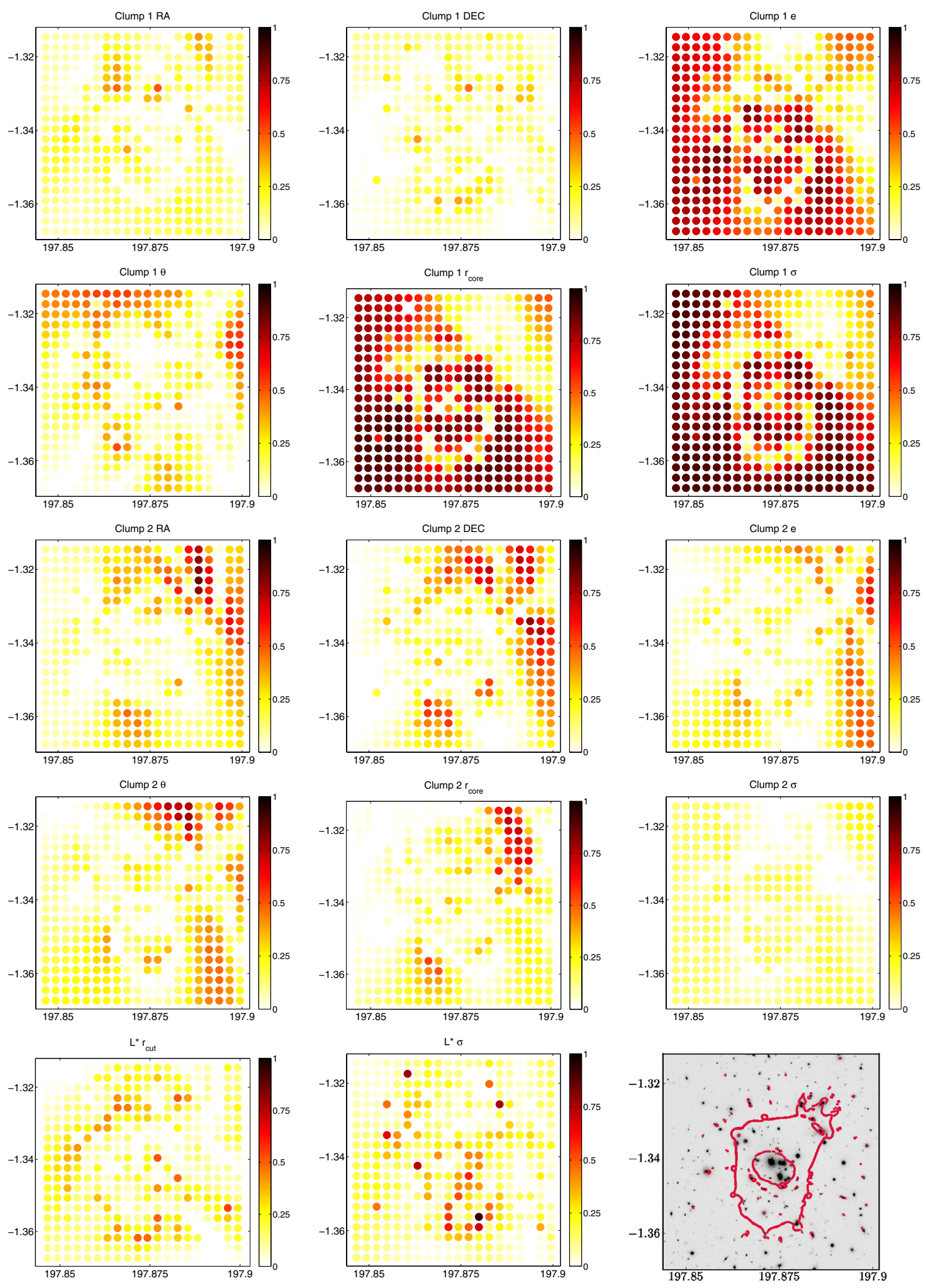

Fig. 3. Correlations between the predicted magnification as a function of image position for a source at redshift $z=2$ and the input parameters that were optimized in the model for A1689 (compare Table 1) in a field of view \pm 100 arcsec around the cluster center. Correlations are given as the absolute values of the correlation coefficients. A high value (close to 1; dark red) indicates that that the magnification for an image at a given position will display large variations when the input parameter is varied. Being able to measure the absolute magification at this image position, using e.g. SNe Ia observations, will thus make it possible to improve the constraints on the cluster parameter. If the correlation is low (close to 0; light yellow), we do not expect magnification information to significantly improve the current constraint on the cluster parameter at hand. The strongest correlations and best possibility of improving the model once a SN Ia is observed, are the ellipticity, $e$, core radius, $r_{\text {core }}$, and velocity dispersion, $\sigma$, of the main lensing potential. The last panel shows an ACS image of the cluster overlaid with the critical lines for $z=2$. The FOV in all panels is identical to that of Fig. 1. 

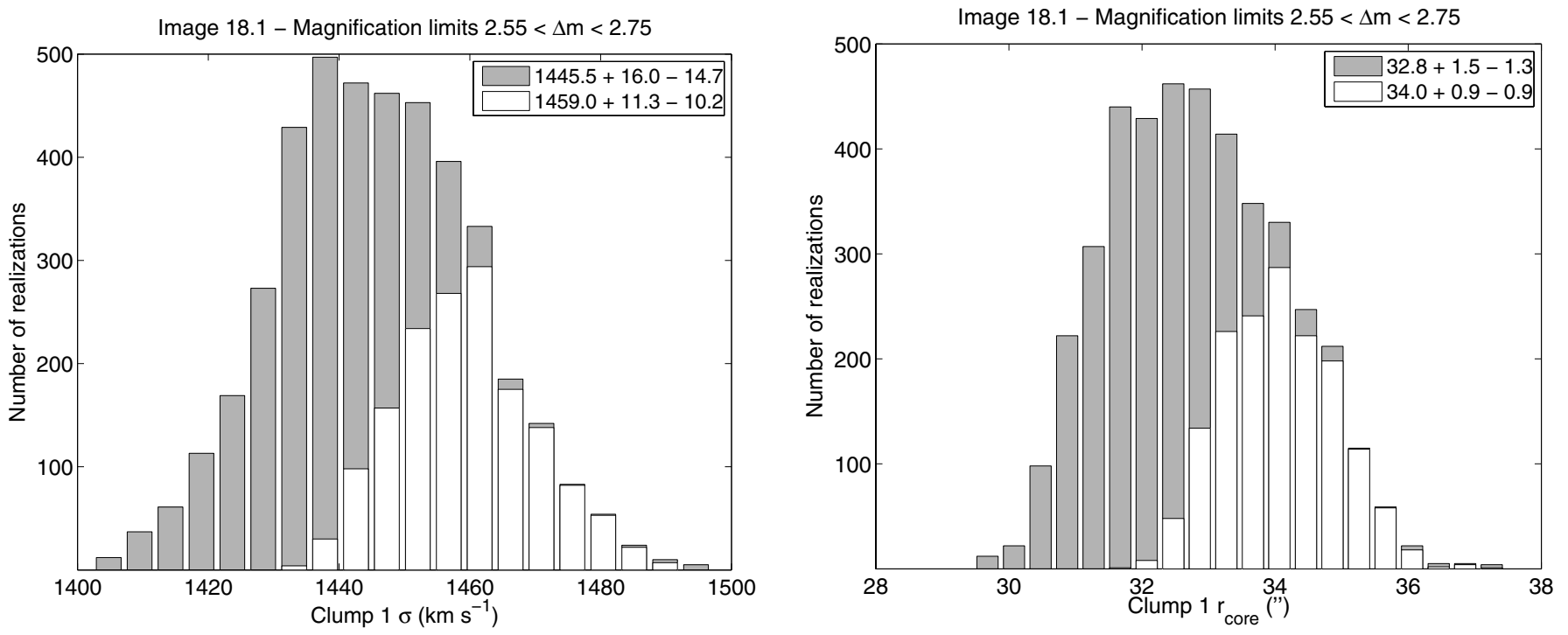

Fig. 4. Possible constraints on the model parameters describing the velocity dispersion (left) and core radius (right) of the main dark matter clump of A1689 using information on the magnification from a SN Ia observed in the multiply lensed background galaxy image 18.1 at $z=1.82$. The shaded bars represent the parameter distribution from all realizations, while the unfilled bars give the parameter distribution considering only realizations, which reproduce the observed magnification within \pm 0.1 mag.

image does not have a counter image with a time delay shorter than five years, it is not included in Table 3. At the position of this image, there are strong correlations between the model parameters and the predicted magnification of this image. The correlation coefficients for the velocity dispersion and core radius of the main clump (clump 1) are 0.84 and 0.87 , respectively. As can be seen in Fig. 4, the possible constraints on $\sigma$ for the main lensing potential improve from $\sim 1 \%$ to $\sim 0.7 \%$, while the corresponding numbers for $r_{\text {core }}$ are $\sim 4.5 \%$ and $\sim 2.6 \%$. Thus, adding only one constraint from the observed magnification of a SN Ia, the constraints on the model parameters for this already very tightly constrained cluster model of A1689 (114 multiple images and 24 spectroscopic redshifts), improve by almost a factor of two. We note that the possible constraints from this image should be seen as a best case scenario, as the correlations for other systems are typically weaker, with median absolute correlation coefficient values in a FOV extending \pm 100 arcsec from the center of the cluster at $z=2$ of 0.67 and 0.71 for the velocity dispersion of the main clump (clump 1) and its core radius, respectively. Since we expect to detect $\sim 5 \mathrm{SNe}$ Ia exploding in all background galaxies per year, we would be able to obtain a combined constraint on these observations of the magnification at several positions behind the cluster.

This technique might prove to be even more interesting for testing the mass modeling of other more poorly constrained galaxy clusters. To evaluate the power of the method, we investigate cluster A2204 with only one known multiply lensed image system, making it much less constrained than A1689, which we have been focusing on so far. Again, we investigate the correlations of the optimized free parameters with the resulting magnifications. The correlation strengths for a grid of image positions \pm 100 arcsec around the cluster center at redshift $z=1$ is shown in Fig. 5. Similarly to the case of A1689, the strongest correlations are found for $\sigma$ and $r_{\text {core }}$ of the main potential with the median absolute correlation coefficient values in the FOV shown in Fig. 5 at redshift $z=1$ being 0.96 and 0.87 , respectively. It is notable that these correlations are rather weak in the central regions of the cluster potential, which is constrained through the location of the known multiple images.
In Fig. 6, we show the constraints on these parameters from the magnification of a hypothetical SN Ia with $z=1$ close to the position of the known lensed image 1.1 measured with an uncertainty of $\pm 0.1 \mathrm{mag}$. The values of the correlation coefficients for $\sigma$ and $r_{\text {core }}$ of the main clump (Clump 1) are 0.90 and 0.79 , respectively. In this case, the constraints on the velocity dispersion for the main lensing potential improve from $\sim 17 \%$ to $\sim 3.5 \%$, while the corresponding constraint for the core radius goes from essentially unconstrained (with a forced upper limit from observations) to $\sim 25 \%$. Thus, observations of SNe Ia behind galaxy clusters promise to be a powerful tool in constraining their overall lens potential and thereby the properties of the dark matter component.

\section{Measuring the Hubble constant}

It was suggested early on that time delays from multiply-lensed sources could be used to measure the Hubble constant (e.g., Refsdal 1964). For a strong lens system, the arrival time of an image at angular position $\boldsymbol{\theta}$ relative to the unlensed case for a corresponding source position $\beta$ is given by

$\Delta t=\frac{1+z_{\mathrm{L}}}{c} \frac{D_{\mathrm{L}} D_{\mathrm{S}}}{D_{\mathrm{LS}}}\left[\frac{(\boldsymbol{\theta}-\boldsymbol{\beta})^{2}}{2}-\psi(\boldsymbol{\theta})\right]$,

where $z_{\mathrm{L}}$ is the redshift of the lens, $D_{\mathrm{L}}, D_{\mathrm{S}}$, and $D_{\mathrm{LS}}$ are, respectively, the angular diameter distances to the lens, to the source, and from the lens to the source, and $\psi(\theta)$ is the lens potential.

Since the ratio of the angular diameter distances scales inversely with the Hubble constant, $D \equiv \frac{D_{\mathrm{L}} D_{\mathrm{S}}}{D_{\mathrm{LS}}} \propto H_{0}^{-1}$. By modeling the lens potential, $\psi(\boldsymbol{\theta})$, and the source position, $\boldsymbol{\beta}$, one can use time delay measurements from strongly lensed systems to estimate the Hubble constant.

There is also a dependence of $D$ on other cosmological parameters, such as $\Omega_{M}$ and $\Omega_{\Lambda}$, although this dependence is much weaker than the dependence of $D$ on the Hubble constant (e.g., Bolton \& Burles 2003; Coe \& Moustakas 2009; Suyu et al. 2010). 

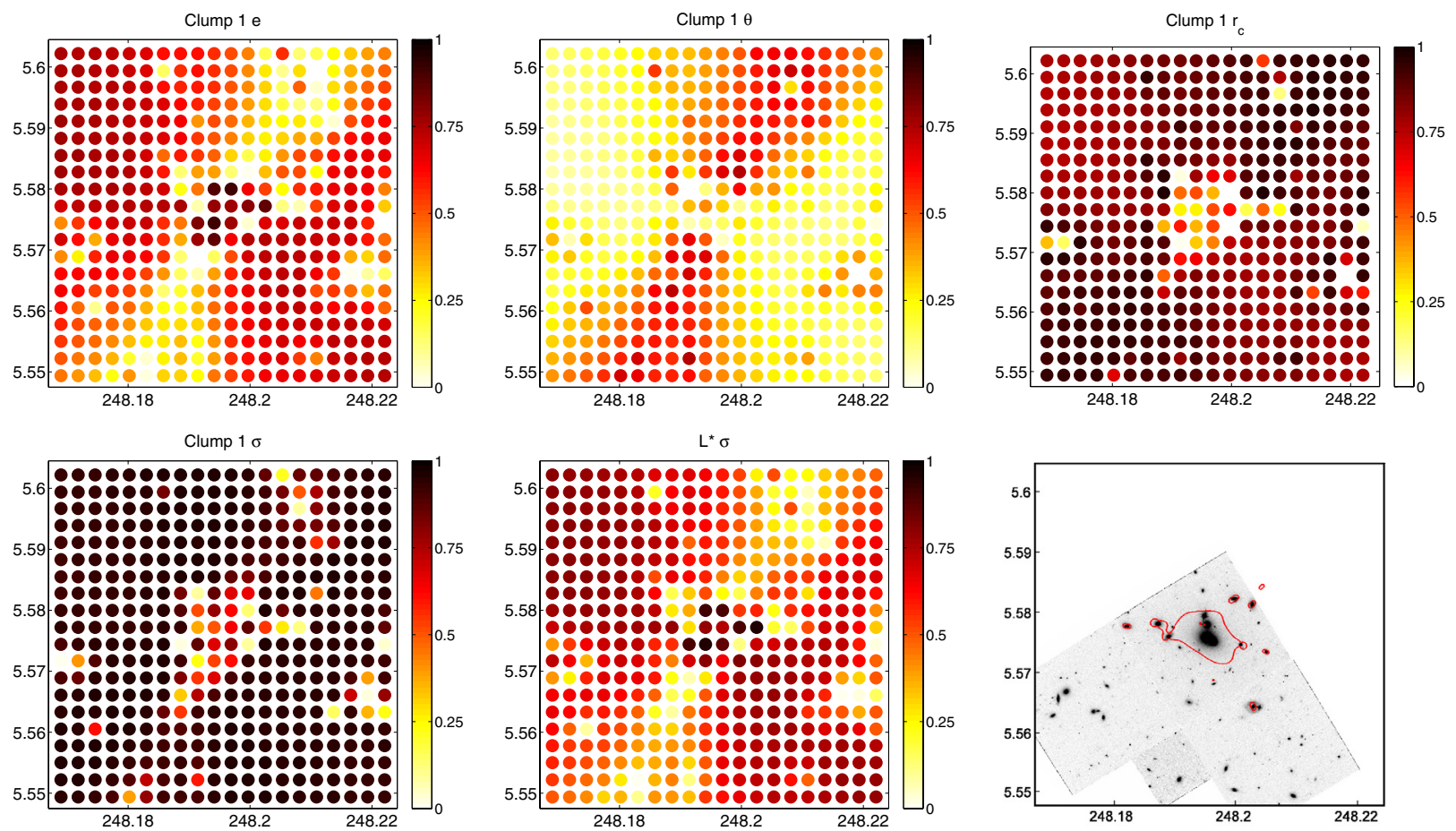

Fig. 5. Correlations between the predicted magnification of an image as a function of image position for a source at redshift $z=1$ and the different input parameters which are optimized in the model for A2204 (compare Table 2 and Fig. 3) in a field of view \pm 100 arcsec around the cluster center. Correlations are given as the absolute values of the correlation coefficients. The last panel shows an ACS image of the cluster overlaid with the critical lines for $z=1$.
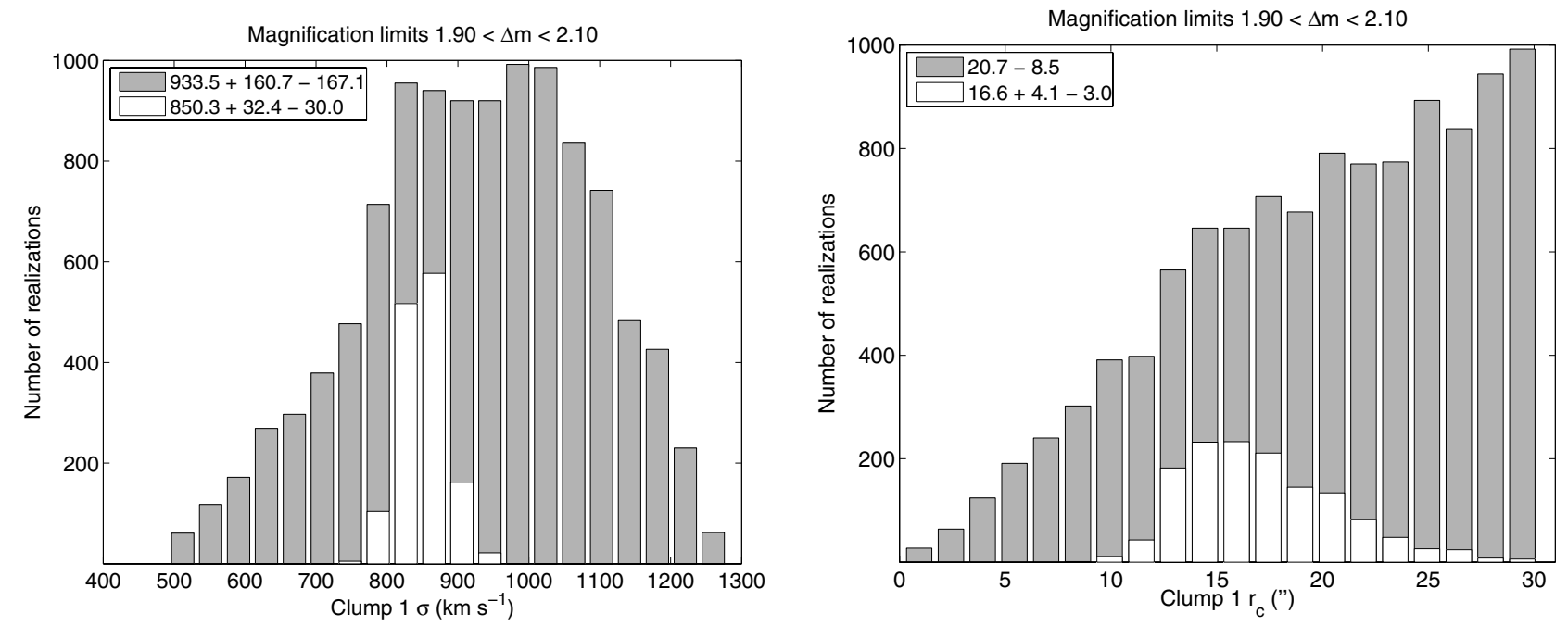

Fig. 6. Possible constraints on the model parameters describing the velocity dispersion (left) and core radius (right) of the main dark matter clump for A2204 using information on the magnification from a SN Ia observed in the image. The shaded bars represent the parameter distribution from all realizations while the unfilled bars give the parameter distribution considering only realizations which reproduce the observed magnification within \pm 0.1 mag.

Owing the transient nature of SNe, the observed time delay in SN light curves can be determined to high precision, typically on the order of less than a few days. To improve the constraints on $H_{0}$ it is thus important to be able to narrow down the errors in the lens potential. Here we investigate the constraints on $H_{0}$ possible when observing a SN in one of the known multiply-imaged background galaxies of A1689 with predicted time delays of shorter than five years and spectroscopic redshifts. Summing up our SN rate estimates (given in Table 3), we expect an average total of three $\mathrm{SNe}$ exploding in those galaxies during a survey time of five years. The effective number of strongly lensed SNe which are observable, will depend on the survey properties. For a five year monthly survey using the HST/WFC3, we would expect to detect approximately one strongly lensed $\mathrm{SN}$ in these galaxies. However, one should keep in mind that there are ten more known strong lensing systems behind A1689, consisting of 30 images, which were not included in this study because they only have photometric redshifts. Furthermore, as observations of this massive cluster continue, additional multiply lensed systems might be discovered (Coe \& Moustakas 2009). 


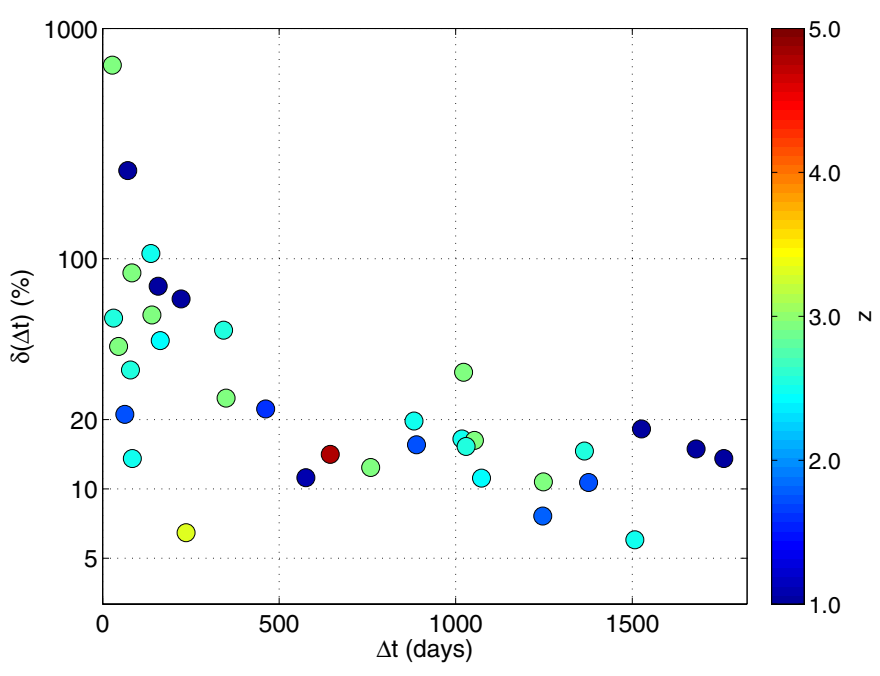

Fig. 7. Predicted time delays in days and $1 \sigma$ confidence interval errors as percentages for all the image pairs of multiply imaged background galaxies with spectroscopic redshifts and time delays shorter than five years behind A1689. The color of the markers indicates the redshifts of the systems.

From the different realizations of the cluster modeling, we get a prediction with error estimates for the time delays between image pairs in the known multiply lensed background galaxies. These errors depend on the positions of the image pairs. As can be seen in Fig. 7, for more than half of the image pairs with predicted time delays shorter than five years and spectroscopic redshifts, the corresponding errors are below 20\%. For several systems, the time delay precision of the model is around $10 \%$ and below. Eventually combining constraints from several strongly lensed $\mathrm{SNe}$, would allow the value of the Hubble constant to be determined to high precision. Thus, nominally, to match the current $\sim 3 \%$ accuracy of the local measurements of $H_{0}$ (Riess et al. 2011), about ten massive clusters should be monitored for five years. Although a rather large investment of telescope time may be required to match a result already at hand, we emphasize the importance of a non-local test of the universal Hubble scale, which would be a key test in cosmology.

In the case of a lensed SN Ia, we can use the information on the absolute magnification to rule out realizations that predict magnifications outside the range allowed by observations. With this extra information, we might be able to more tightly constrain the time delay error of the lens model. Therefore, we check for correlations between the predicted magnification of the images in a system and the time delay between the images. While many systems display no or only weak correlations between magnifications and time delay, there have been more promising results for a few other systems.

Figure 8 shows an example of the cuts in time delay error that can be made using magnification constraints from a lensed SN Ia in background galaxy 19. In this case, the errors in the time delay between images 19.3 and 19.4 can be reduced from $\approx_{-12.5}^{+15.1}$ to $\approx_{-9.7}^{+11.4} \%$.

As discussed in Sect. 4, the additional model constraints provided by a measurement of the magnification based on the standard candle nature of SNe Ia are only modest for a tightly constrained cluster mass model such as that of A1689. However, this constraint would prove far more valuable in the case of a time delay measured in a more weakly constrained cluster mass model as it could break the degeneracy between the mass profile and the Hubble constant (Oguri \& Kawano 2003).

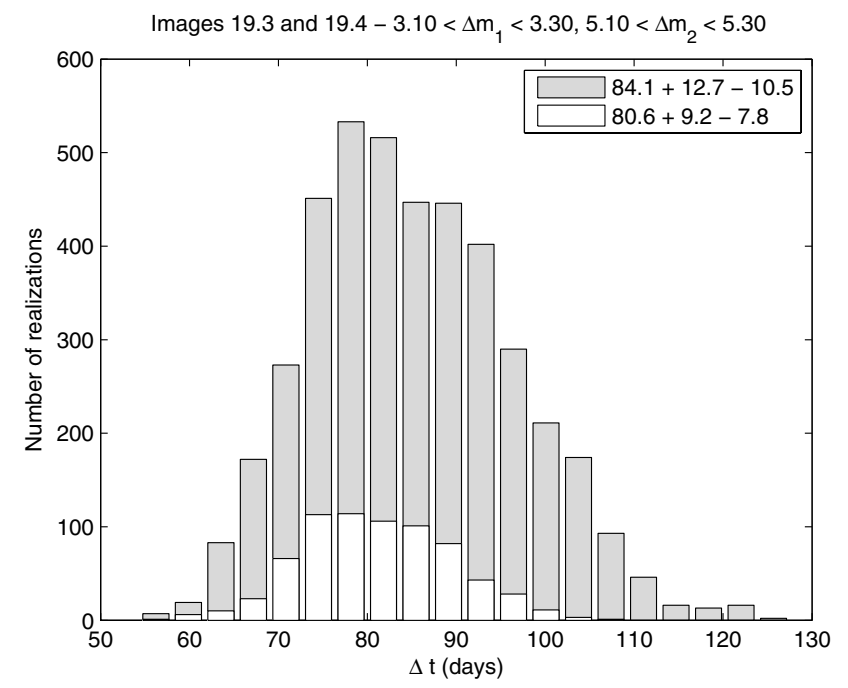

Fig. 8. Constraints on the time delay between images 19.3 and 19.4 at $z=2.60$ behind A1689 using the magnification of both images. In the shaded histogram we show the time delay distribution from the complete Monte Carlo Markov chain. The unfilled histogram indicates the distribution after the cuts corresponding to magnification constraints. Errors are given as $1 \sigma$ confidence intervals.

\section{Discussion and conclusions}

A SN Ia exploding in any of the background galaxies of a cluster can provide give an absolute measure of the magnification at that point and therefore potentially be used to constrain the mass modeling of the cluster. For cluster A1689, which we have focused on in this study, the cluster potential had previously been carefully modeled. However, this additional constraint from one or more lensed SNe could help us to improve the cluster model further by reducing the parameter uncertainties. For galaxy clusters with more weakly constrained mass profiles, this technique is even more interesting. Using the magnification information from a single SN Ia behind cluster A2204 can be a powerful tool to narrow down the model parameters of the dark matter halo component.

Since $\mathrm{SNe}$ are point-like sources for a limited period, microlensing from stars in a cluster galaxy close to the line of sight might in principle significantly affect the magnification and thus the possible constraints on the large-scale mass model from SNe Ia. However, it has been concluded (Oguri \& Kawano 2003) that this effect should only cause small deviations. Microlensing events caused by massive compact halo objects (MACHOs) in the intracluster medium might unambiguously be identified in SN light curves. Thus, any modulation of the light curve shape, or lack thereof, can place limits on the mass fraction of the cluster mass in the form of $10^{-7}<M / M_{\odot}<10^{-4}$ MACHOs (Kolatt \& Bartelmann 1998).

In the case of a SN exploding in one of the known multiply lensed background galaxies, there is a good chance of observing multiple images of the SN and determining the observed time delay with high precision. The intrinsically brighter SNe, e.g. SNe Ia and SNe IIn, should in principle be detectable in essentially all of the systems. However, for SNe IIP, which are expected to be the most common SN type, the magnification is only strong enough for them to become observable in about half of the cases.

Owing the well-constrained mass model for galaxy cluster A1689, SN time delays can be used as an independent measure on the Hubble parameter at high redshift. In the case of a SN Ia, 
the magnification might also be used as a constraint to reduce the uncertainty in the time delay predicted by the lensing model. Another advantage of using a cluster with a well-constrained mass model is the possibility of predicting subsequent images when a $\mathrm{SN}$ behind the cluster is observed.

It is quite exciting that CLASH, a cluster monitoring multicycle program, is currently been pursued (Postman et al. 2011). However, the strategy in the CLASH program is tuned to finding and accurately studying $\mathrm{SNe}$ Ia in the parallel fields, i.e., in the low magnification region. Much of the observations of the cluster cores are performed in optical and UV filters, where high- $z \mathrm{SNe}$ would not be detectable. Furthermore, the cluster fields are only monitored for 2-4 months making the search for multiple images practically impossible, especially considering the time dilation $(1+z)=2-4.5$ in the lensed SN lightcurves.

Corroborating the value of $H_{0}$ found in the local universe could provide crucial support to our cosmological picture. However, any discrepancy would falsify the accepted scenario and shed new light into the nature of both dark matter and dark energy. Since strongly lensed SNe involve both angular diameter and luminosity distances $\left(d_{\mathrm{A}}\right.$ and $\left.d_{\mathrm{L}}\right)$, these systems provide a unique opportunity to probe the distance reciprocity relation, $d_{\mathrm{L}}=(1+z)^{2} d_{\mathrm{A}}$. Any violation of this relation would indicate either the existence of an unaccounted for astrophysical systematic effect affecting one of the distance measure but not the other, or that the Universe is not described by the standard cosmological model. Specifically, a violation could indicate that the Universe is described by a non-metric theory of gravity or a theory in which light does not travel on unique null geodesics. Current tests of $d_{\mathrm{L}} / d_{\mathrm{A}}$ are restricted to $z<1$ (e.g., Lampeitl et al. 2010) and have $>20 \%$ uncertainties.

Acknowledgements. E.M. would like to thank the Swedish Research Council for financial support. The Dark Cosmology Centre is funded by the Danish National Research Foundation.

\section{References}

Amanullah, R., Goobar, A., Clément, B., et al. 2011, ApJ, 742, L7

Bell, E. F., McIntosh, D. H., Katz, N., \& Weinberg, M. D. 2003, ApJS, 149, 289

Bolton, A. S., \& Burles, S. 2003, ApJ, 592, 17

Bradač, M., Treu, T., Applegate, D., et al. 2009, ApJ, 706, 1202

Bradley, L. D., Bouwens, R. J., Ford, H. C., et al. 2008, ApJ, 678, 647

Broadhurst, T., Benítez, N., Coe, D., et al. 2005, ApJ, 621, 53

Casali, M., Pirard, J.-F., Kissler-Patig, M., et al. 2006, SPIE, 6296, 29

Coe, D., \& Moustakas, L. 2009, ApJ, 706, 45

Conley, A., Guy, J., Sullivan, M., et al. 2011, ApJS, 192, 1

Dahlén, T., Mobasher, B., Dickinson, M., et al. 2007, ApJ, 654, 172

Dawson, K. S., Aldering, G., Amanullah, R., et al. 2009, AJ, 138, 1271

Fohlmeister, J., Kochanek, C. S., Falco, E. E., Morgan, C. W., \& Wambsganss, J. 2008, ApJ, 676, 761

Goobar, A., \& Leibundgut, B. 2011, Annu. Rev. Nucl. Part. Sci., 61, 251

Goobar, A., Mörtsell, E., Amanullah, R., \& Nugent, P. 2002, A\&A, 393, 25

Goobar, A., Paech, K., Stanishev, V., et al. 2009, A\&A, 507, 71 (Paper II)

Inada, N., Oguri, M., Morokuma, T., et al. 2006, ApJ, 653, L97

Jullo, E., Kneib, J.-P., Limousin, M., et al. 2007, NJPh, 9, 447

Kissler-Patig, M., Pirard, J.-F., Casali, M., et al. 2008, A\&A, 491, 941

Kneib, J.-P., Ellis, R. S., Santos, M. R., \& Richard, J. 2004, ApJ, 607, 697

Kochanek, C. S. 2002, ApJ, 578, 25

Kolatt, T., \& Bartelmann, M. 1998, MNRAS, 296, 763

Lampeitl, H., Nichol, R. C., Seo, H.-J., et al. 2010, MNRAS, 401, 2331

Limousin, M., Richard, J., Jullo, E., et al. 2007, ApJ, 668, 643

Moorwood, A., Cuby, J.-G., Biereichel, P., et al. 1998, The Messenger, 94, 7

Mörtsell, E., \& Sunesson, C. 2006, JCAP, 01, 012

Oguri, M., \& Kawano, Y. 2003, MNRAS, 338, 25

Pirard, J.-F, Kissler-Patig, M., Moorwood, A., et al. 2004, SPIE, 5492, 1763

Oguri, M. 2007, ApJ, 660, 1

Postman, M., Coe, D., Benitez, N., et al. 2011, ApJS, accepted [arXiv: 1106.3328]

Refsdal, S. 1964, MNRAS, 128, 307

Richard, J., Smith, G. P., Kneib, J.-P., et al. 2010, MNRAS, 404, 325

Riess, A. G., Macri, L., Casertano, S., et al. 2011, ApJ, 732, 129

Scannapieco, E., \& Bildsten, L. 2005, ApJ, 629, L85

Stanishev, V., Goobar, A., Paech, K., et al. 2009, A\&A, 507, 61 (Paper I)

Suyu, S. H., Marshall, P. J., Auger, M. W., et al. 2010, ApJ, 711, 201

Wambsganss, J., \& Paczynski, B. 1994, AJ, 108, 1156

Witt, H., Mao, S., \& Keeton, C. 2000, ApJ, 544, 98

Zhao, H., \& Qin, B. 2003, ApJ, 582, 2

Zheng, W., Bradley, L. D., Bouwens, R. J., et al. 2009, ApJ, 697, 1907 